\title{
Electrical Conductivity of Melt Compounded Functionalized Graphene Sheets Filled Polyethyleneterephthalate Composites
}

\author{
Haobin Zhang ${ }^{1}$, Shunlun $\mathrm{He}^{2 *}$, Cao Chen ${ }^{1}$, \\ Wenge Zheng ${ }^{1 *}$ and Qing Yan ${ }^{1}$ \\ ${ }^{1}$ Ningbo Key Laboratory of Polymer Materials, Division of Polymers and Composites, \\ Ningbo Institute of Material Technology \& Engineering, Chinese Academy of Sciences, \\ ${ }^{2}$ Orinko Plastics Group, \\ China
}

\section{Introduction}

Graphene, monolayer of carbon atoms arranged in a honeycomb network, has recently gained revolutionary aspirations (Novoselov et al., 2005; Novoselov et al., 2007; Heersche et al., 2007; Zhang (a) et al., 2005; Stankovich et al., 2006) because of its remarkable electronic properties (Zhang (b) et al., 2005; Berger et al., 2004), unusual thermal properties (Balandin et al., 2008) and good mechanical properties (Lee et al., 2008). These extraordinary properties make it an excellent choice as inorganic fillers to substantially improve electrical, thermal and mechanical properties of composite materials (Stankovich et al., 2006; Ramanathan et al., 2008). Several effective techniques have been developed for preparing graphene nanosheets, including chemical (Stankovich et al., 2006) and mechanical exfoliation (Novoselov et al., 2004), alkali metals intercalation and expansion (Viculis et al., 2003), microwave chemical vapor deposition (Wang et al., 2009), substrate-based thermal decomposition (Berger et al., 2004), and thermal exfoliation of graphite oxide (GO) (McAllister et al., 2007). Among them, chemical reduction of exfoliated graphite oxide in the presence of a surfactant or polymer is a relatively new method to prepare electrically conductive individual graphene sheets. Stankovich (Stankovich et al., 2006) put forward a new process to produce polystyrene/graphene nanocomposites via ultrasonic exfoliation and chemical reduction of graphite oxide and molecular-level dispersion of chemically modified graphene nanosheets. In addition, the thermal exfoliation and in situ reduction method can conveniently produce graphene nanosheets for mass production. As confirmed by Aksay and co-workers (McAllister et al., 2007), in situ reduction reaction took place during the thermal exfoliation process, which converted insulating graphite oxide to conducting graphene. More importantly, the graphene resulted through thermal exfoliation still contained some oxygen-containing groups. The oxygen functionalities on the graphene nanosheets will facilitate their dispersion in polar polymers (Ramanathan et al., 2008). Effective medium approximation indicated that graphene is more effective in improving conductivity of composites than carbon nanotubes (Xie et al., 2008). The polystyrene/graphene nanocomposites prepared by chemical modification and reduction in 
solution exhibited a percolation threshold in electrical conductivity as low as $0.1 \mathrm{vol} \%$, comparable to those observed in single-walled carbon nanotubes (SWCNT) based nanocomposites (Grossiord et al., 2005; McLachlan et al., 2005); The electrical conductivity was as high as $1 \mathrm{~S} / \mathrm{m}$ at $2.5 \mathrm{vol} \%$ of graphene content. Kim and Macosko (Kim \& Macosko, 2008) compared the effect of graphene nanosheets and graphite on conductivity of polyester-based composites, and the results revealed that graphene gave a much lower percolation threshold $(0.3 \mathrm{vol} \%)$ than graphite $(3.0 \mathrm{vol} \%)$. Recently, Liang et al. (Liang et al., 2009) prepared epoxy/graphene nanocomposites via in situ process, which showed a small percolation threshold of $0.52 \mathrm{vol} \%$ and an electromagnetic interference shielding efficiency of $21 \mathrm{~dB}$ with $8.8 \mathrm{vol} \%$ of graphene over $8.2 \sim 12.4 \mathrm{GHz}$. In contrast, unfully exfoliated graphite led to a much higher threshold $(>0.6 \mathrm{vol} \%)$ and a much lower electrical conductivity $(<10-2 \mathrm{~S} / \mathrm{m}$ ) even at high loading of $6.0 \mathrm{vol} \%$ (Kalaitzidou et al., 2007; Weng et al., 2005; Zheng et al., 2002).

Compared to in situ polymerization and solution mixing, melt compounding using conventional compounding devices such as mixers and commercial resins is more attractive because this approach provides manufacturers many degrees of freedom with regard to the selection of polymer grades and choice of graphene content. It is believed that melt compounding would be more economical and simple than in situ polymerization processes. Actually, melt compounding has been successfully used to prepare conductive polymeric composites by using conducting fillers such as carbon nanotubes (CNTs) (Li et al., 2006), carbon black and expanded graphite (Lagreve et al., 2006; Zhang et al., 2008). It has been confirmed that CNTs can substantially increase the electrical conductivity of PET nanocomposites with low loading of CNT, but it is expensive. As for cheap carbon black, higher loading is usually required to make a polymer conductive. Due to the low price and availability of pristine graphite in large quantities, graphene nanosheets can be an ideal choice as conductive fillers in the preparation of conductive PET nanocomposites. To the best of our knowledge, few papers have been published on PET/graphene nanocomposites prepared by conventional melt compounding. In this study, Graphene nanosheets were prepared by complete oxidation of pristine graphite followed by thermal exfoliation and reduction. Subsequently, polyethylene terephthalate/graphene nanocomposites were prepared by melt compounding. The microstructure of graphene, its dispersion in PET matrix, and the electrically conductive behavior of the resulting nanocomposites were studied.

\section{Experimental}

\subsection{Materials}

PET pellets were purchased and dried in vacuum oven at $150 \mathrm{oC}$ for $5 \mathrm{~h}$ before use. Flaky pristine graphite with a mean size of $45 \mu \mathrm{m}$ was bought from Qingdao Huatai Lubricant Sealing S\&T (China). Graphene density is assumed to be equal to the theoretical graphite density of $2.28 \mathrm{~g} / \mathrm{cm} 3$ (Kelly, 1981), and PET density is $1.34 \mathrm{~g} / \mathrm{cm} 3$ (Cobbs \& Burton, 1953). Fuming nitric acid (63\%), sulfuric acid (98\%), potassium chlorate $(98 \%)$ and hydrochloric acid (37\%) were obtained from Sinopharm Chemical Reagent (China).

\subsection{Preparation of graphene}

A technique, similar to that of Aksay and co-workers (Schniepp et al., 2006), was used to prepare bulk quantities of graphene nanosheets. Graphite oxide (GO) was prepared according to Staudenmaier method (Staudenmaier, 1898). First of all, the reaction flask was 
purged with nitrogen and immersed in an ice bath, $40 \mathrm{~g}$ graphite was then added to the homogeneous mixture of concentrated nitric acid $(270 \mathrm{ml})$ and sulfuric acid $(525 \mathrm{ml})$ under vigorous stirring. After uniform dispersion of the graphite powder, $330 \mathrm{~g}$ potassium chlorate was added slowly to minimize the risk of explosion. The reaction was then allowed to last for $120 \mathrm{~h}$ at room temperature. After reaction, the slurry resultants were filtered and washed with excess deionized water and $5 \% \mathrm{HCl}$ solution to remove the sulfuric ions $\left(\mathrm{SO}_{4}{ }^{2-}\right.$ ), then GO aqueous solution was neutralized with potassium hydroxide solution. GO powder was separated from the solution by using GQ75 high-speed centrifuge, and dried in an air-circulating oven at $135^{\circ} \mathrm{C}$ for $24 \mathrm{~h}$ followed by another $24 \mathrm{~h}$ at $135^{\circ} \mathrm{C}$ in a vacuum oven. As confirmed by Aksay and co-workers (McAllister et al., 2007), $1050^{\circ} \mathrm{C}$ was adequate for thermal exfoliation and in situ reduction of GO, this temperature was also adopted in the present work to thermally exfoliate GO. The dried GO powder was quickly inserted into a muffle furnace preheated to $1050 \mathrm{oC}$ and held in the furnace for 30s.

\subsection{Fabrication of PET composites}

PET/graphene nanocomposites were prepared by melt compounding at $285^{\circ} \mathrm{C}$ using a Brabender mixer. Compounding was performed with an initial screw speed of $50 \mathrm{rpm} / \mathrm{min}$ for 4 minutes; then the screw speed was raised to $100 \mathrm{rpm} / \mathrm{min}$ within $1 \mathrm{~min}$ and the compounding was conducted at this speed for $5 \mathrm{~min}$. The samples for microscopy and electrical conductivity analysis were prepared by pressing the composites at $275^{\circ} \mathrm{C}$ under a pressure of $15 \mathrm{MPa}$.

\subsection{Characterization}

The volume conductivity of moderately conductive composites (Conductance $>10^{-6} \mathrm{~S} \mathrm{~m}$ ) was measured with a Agilent 34401A digital multimeter and a YOKOGAWA 7651 voltage source by the two-probe method. For PET and composites with low conductivity (Conductance $\leq 10^{-6} \mathrm{~S} \mathrm{~m}$ ), the conductivity testing was performed using a EST121 ultrahigh resistance and micro current meter (Beijing EST Science \& Technology CO. LTD) according to National Standard of China GB/1410-1989. A three-terminal fixture (two electrodes plus guard), similar to that proposed by the ASTM D257 standard for volume conductance determination of flat specimens, was selected for the measurement of low conductance. The guard electrode connected to the earth was used to eliminate the leakage current along specimen surface. In addition, the testing fixture was embedded in a metal shield box to minimize external interference. Circular plates with $7 \mathrm{~cm}$ in diameter and $2.5 \mathrm{~mm}$ in thickness were fabricated for conductivity measurement. Sample surfaces were coated with silver paste to reduce contact resistance between sample and electrodes. For specimens with low conductivity, the side contacting with the unguarded electrode was wholly painted with silver paste while the other side only partly painted, the painted area was in accordance with the cross-section of the guarded cylinder electrode. Three specimens of each sample were tested and the average value was reported as volume electrical conductivity. The resistance $\mathrm{R}$ can be obtained directly from the multimeter, and thus the electrical conductivity o can be calculated by using Eq. (1)

$$
\sigma=\frac{1}{\rho}=\frac{\mathrm{t}}{R S}
$$

where $\rho$ is electrical resistivity, $t$ is thickness of sample between electrodes, and $S$ is crosssectional area of the guarded cylinder electrode. 
The microstructures of graphene and its PET nanocomposites were characterized using a Tecnai G2 F20 TEM at an accelerating voltage of $100 \mathrm{kV}$. Graphene sheets were dispersed in methanol by ultrasonication and some pieces were collected on carbon-coated 300-mesh copper grids for observation. While for PET/graphene nanocomposites, the samples were embedded in epoxy resin, and cured at $80 \mathrm{oC}$ for $6 \mathrm{~h}$, and then ultrathin sections of thinner than $100 \mathrm{~nm}$ were cryogenically cut with a diamond knife using a microtome and collected on 300-mesh copper grids. SEM observation of PET composites was performed using an FEI scanning electron microscope (Hitachi, S-4800) with an accelerating voltage of $5 \mathrm{kV}$. PET composites were freeze-fractured in liquid nitrogen and the fractured section was coated with a thin layer of platinum before observation.

The diffraction behavior of pristine graphite, GO and graphene were studied using a Bruker AXS X-ray diffractometer with CuKa radiation at a generator voltage of $40 \mathrm{kV}$ and a generator current of $40 \mathrm{~mA}$. The scans were carried out in the reflection mode at a scan rate of $10^{\circ} / \mathrm{min}$. Laser granularity analyzer (Malven, Zeta sizer Nano ZS) was used to determine the lateral dimension of graphene in alcohol solution.

\section{Results and discussion}

\subsection{Characterization of graphene}

Figure. 1 shows the XRD spectra of pristine graphite, graphite oxide and graphene. The strong and sharp diffraction peak of pristine graphite at 26.6o completely disappeared after oxidization and instead a new peak at $13.9^{\circ}$ appeared, indicating a complete oxidization of graphite (Wang et al., 2008), which is a prerequisite to obtain exfoliated graphene nanosheets by ultrasonication or thermal expansion. After thermal exfoliation of the completely oxidized graphite (GO), there was no apparent diffraction peak detected, which means the periodic structure of GO was eliminated and graphene nanosheets were formed.

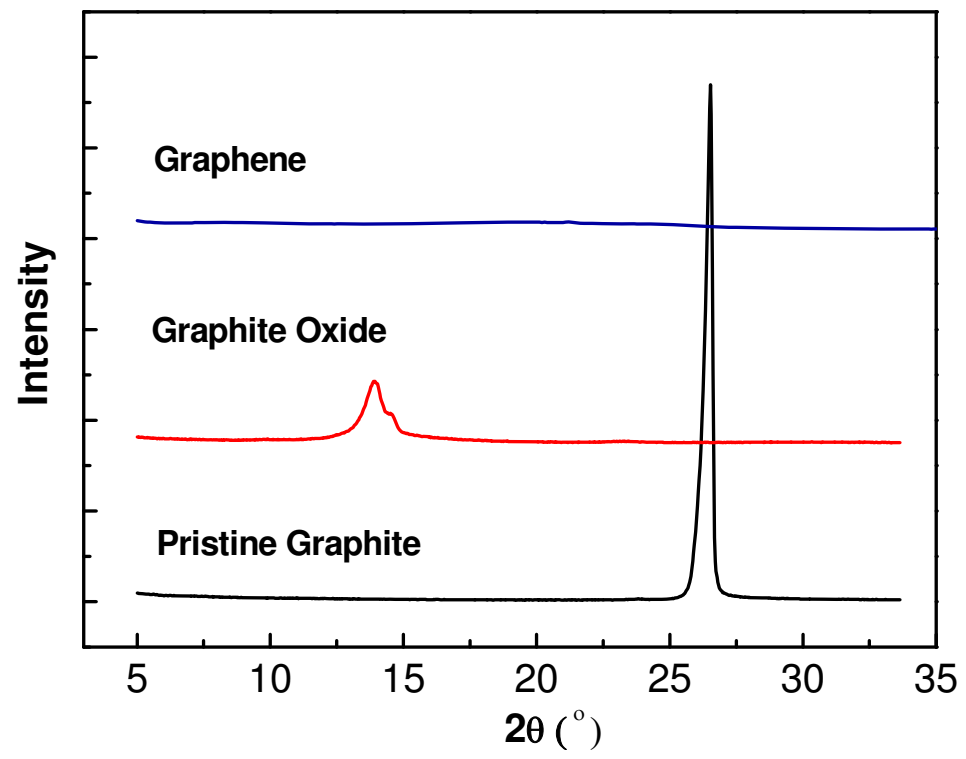

Fig. 1. X-ray diffraction patterns of pristine graphite, graphite oxide and graphene. 
Figure 2 shows TEM images of the graphene under low and high magnifications. Under low magnification, graphene looks like a transparent ultrathin film with a few thin ripples within the plane (Figure. 2a), quite different from the opaque and smooth feature of pristine graphite flakes. It is known that perfect two-dimensional graphene crystal was thermodynamically unstable (Meyer et al., 2007), therefore corrugations and ripples in the two dimension were formed for thermodynamic stability (Carlsson, 2007). Thereby the transparency and rippled feature of graphene, in turn, suggests a fact that graphene sheets prepared in this study are wafer thin and consists of few-layer or even monolayer graphene. Furthermore, stacks of graphene sheets arranged with a roughly parallel structure can be clearly observed in the high-magnification TEM image from an edge-on view (Figure. 2b).

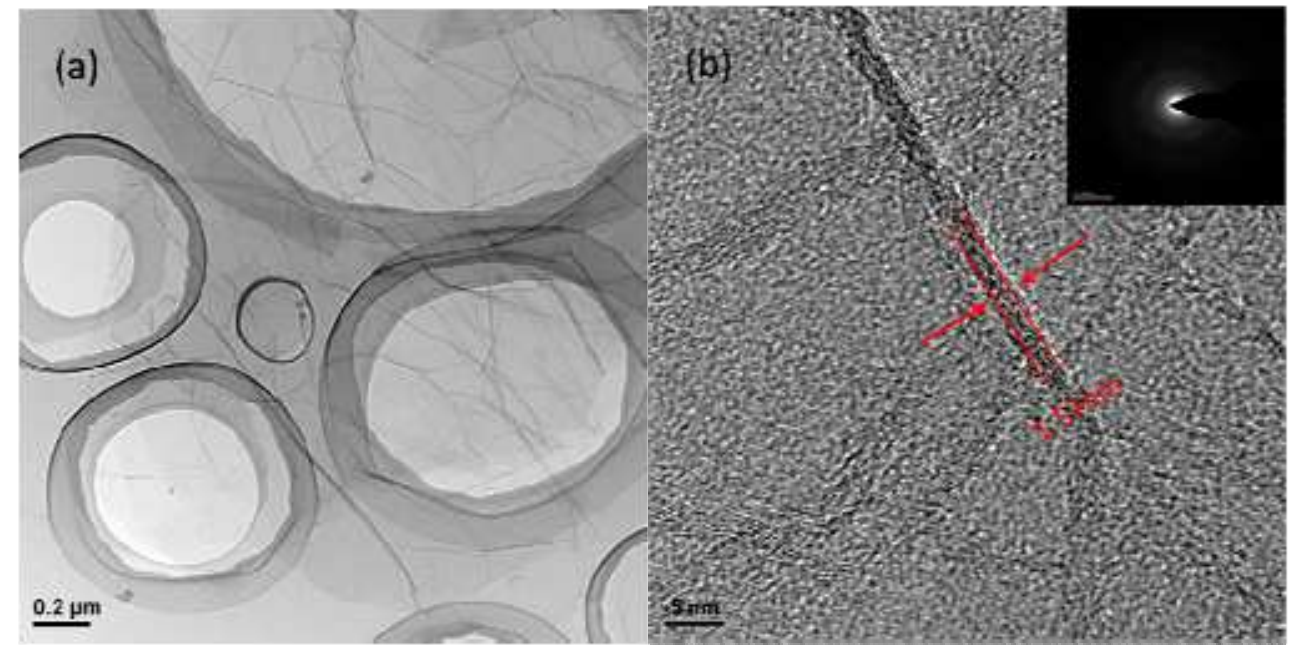

Fig. 2. TEM images of graphene under low (a) and high (b) magnifications. The scale bars are (a) $0.2 \mu \mathrm{m}$ and (b) $5 \mathrm{~nm}$. The inset is selected area electron diffraction (SAED) pattern of graphene.

The thickness of graphene sheets can thus be measured. Statistical results from many TEM images give an average thickness $\sim 1.57 \mathrm{~nm}$ of the graphene sheets prepared in this study. In addition, the weak and diffuse selected area electron diffraction (SAED) pattern (the inset of figure. $2 b$ ) also indicates the loss of long range ordering between graphene sheets and the ultrathin feature of the graphene. However, it should be mentioned that the specific surface area of the graphene sheets measured with BET method is $555 \mathrm{~m} 2 / \mathrm{g}$, much smaller than the theoretical value $(2630 \mathrm{~m} 2 / \mathrm{g})$ of single graphene sheet (Peigney et al., 2001), indicating that existence of agglomerated and overlapped parts of the graphene sheets. In spite of this, a high aspect ratio (lateral dimension to thickness) of 146 for the graphene sheets is obtained based on the results from laser granularity analyzer. In contrast, pristine graphite flake has a lower aspect ratio $(\sim 36)$ and smaller specific surface area $(<10 \mathrm{~m} 2 / \mathrm{g})$.

\subsection{Microstructure of PET/graphene nanocomposites}

To clearly reveal the dispersion of graphene in PET matrix, Figure 3 shows the microstructure of PET nanocomposite with 3 vol\% graphene. Graphene nanosheets were 


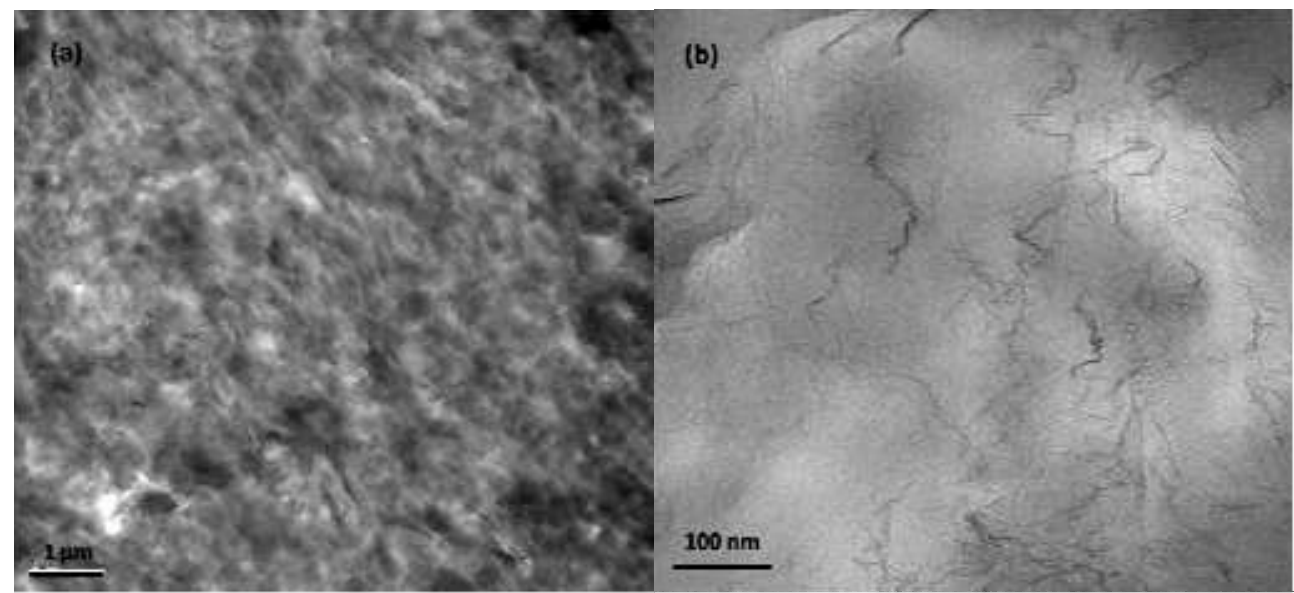

Fig. 3. Low (a) and high (b) magnification TEM micrographs of PET nanocomposite with 3 vol\% graphene. The scale bars are (a) $1 \mu \mathrm{m}$ and (b) $100 \mathrm{~nm}$.

homogeneously dispersed in PET matrix and there are almost no large agglomerates observed (Figure. 3a).

The good dispersion of graphene sheets should be due to the good interaction between the oxygen and hydroxyl functional groups on the surface of graphene and the polar groups of PET. In addition, a compact continuous network throughout the matrix is also observed (Figure. 3a). As proposed by Alig et al (Alig et al., 2007), this conductive network can be considered as a network built by conductive fillers which are separated by local contact regions with polymer chains in-between, also improving electron mobility and interface polarization via tunneling (Yao et al., 2007). High magnification image (Figure. 3b) indicates that the network is composed of abundant thin stacks of a few sheets of monolayer graphene (black curly thin lines), which are bridged by the crumpled or overlapped graphene sheets (slightly thicker lines) (Figure. 3b). These wrinkled and overlapped graphene sheets can effectively link individual graphene sheets and carry high density of current, resulting in high electrical conductivity (Halperin et al., 1985).

To demonstrate the strong interaction between the oxygen and hydroxyl functional groups on the surface of graphene and the polar groups of PET, Figure 4 shows SEM image of cryofractured surface of PET nanocomposite with $1.2 \mathrm{vol} \%$ graphene. It is seen that the graphene sheets were encapsulated by PET and the thickness of the sheets increased from 1.57 to $~ 50$ $\mathrm{nm}$ (see the inset of Fig. 4). This confirms the strong interaction between graphene and PET. Due to the large specific surface area of the graphene nanosheets, the area of interface between graphene and PET is huge, providing numerous tunneling sites for electron transport (Steinert \& Dean, 2009).

\subsection{Electrical properties of PET/graphene nanocomposites}

Figure. 5 shows plots of electrical conductivity versus filler content for PET/graphene nanocomposites and PET/pristine graphite composites.

The PET/graphene nanocomposites exhibit a sharp transition from insulator to semiconductor with a small percolation threshold of $0.47 \mathrm{vol} \%$. With a small increase of graphene content from 0.47 to $1.2 \mathrm{vol} \%$, the electrical conductivity of the PET 


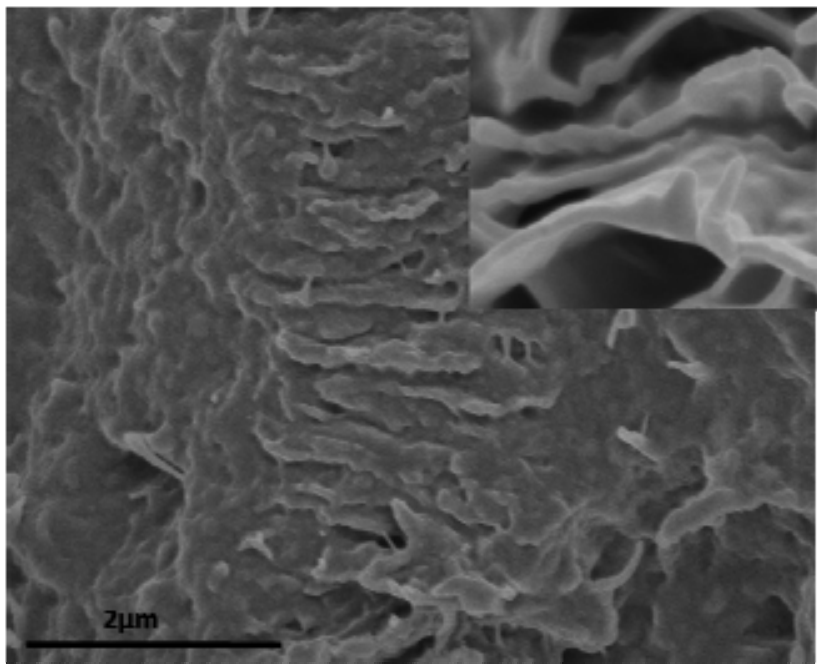

Fig. 4. SEM images of fractured surface of PET nanocomposite with $1.2 \mathrm{vol} \%$ of graphene. The insert is a high-magnification SEM image of the same nanocomposite.

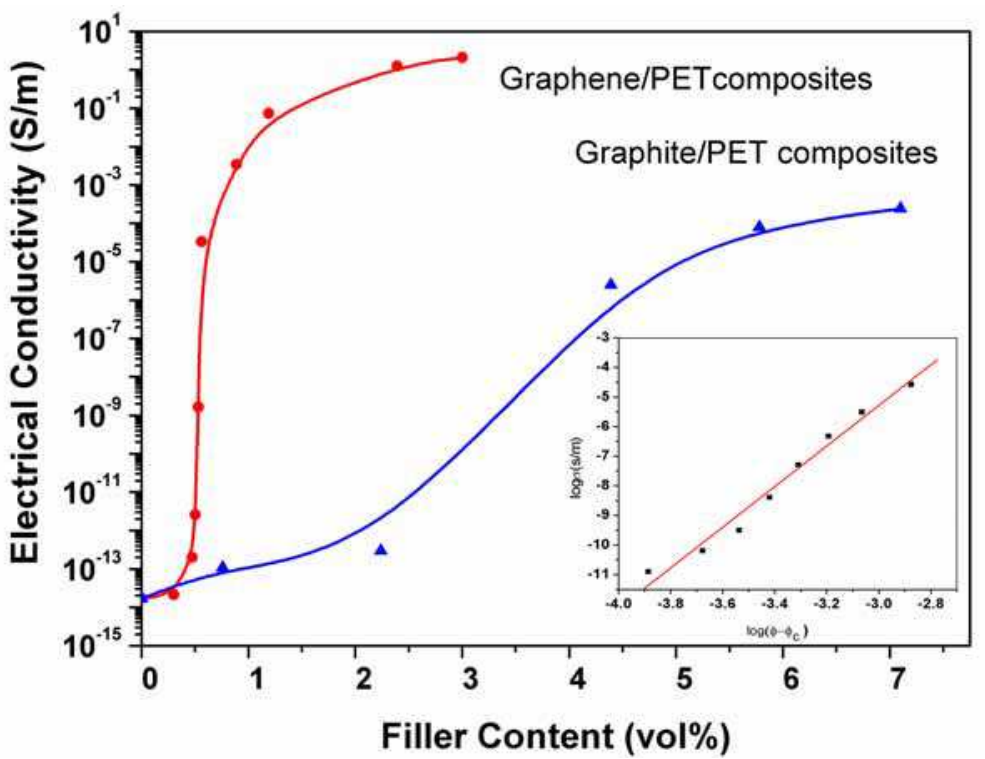

Fig. 5. Plots of electrical conductivity versus filler content for PET/graphene nanocomposites and PET/graphite composites. The inset is double-logarithmic plot of volume electrical conductivity versus $(\varphi-\varphi c)$.

nanocomposites increased quickly from $2.0 \times 10^{-13}$ to $7.4 \times 10^{-2} \mathrm{~S} / \mathrm{m}$. Actually, at $0.56 \mathrm{vol} \%$ of the graphene content, the conductivity the PET nanocomposite is $3.3 \times 10^{-5} \mathrm{~S} / \mathrm{m}$, which is higher than antistatic criterion of $10^{-6} \mathrm{~S} / \mathrm{m}$. With only $3.0 \mathrm{vol} \%$ graphene, the conductivity of 
PET nanocomposite is as high as $2.11 \mathrm{~S} / \mathrm{m}$. On the contrary, PET/graphite composites show a much higher percolation threshold of $2.4 \mathrm{vol} \%$ and a very broad percolation transition within a range of graphene content from 2.4 to $5.8 \mathrm{vol} \%$. More interestingly, graphene is more efficient in improving electrical conductivity of PET than pristine graphite. For instance, the conductivity of PET/graphene nanocomposite with $0.92 \mathrm{vol} \%$ of graphene is $3.46 \times 10^{-3} \mathrm{~S} / \mathrm{m}$, even higher than that of PET/pristine graphite composite with $7.1 \mathrm{vol} \%$ of graphite $\left(2.45 \times 10^{-4} \mathrm{~S} / \mathrm{m}\right)$.

According to the percolation theory (Stauffer, 1985), the relationship of composite conductivity $(\sigma)$ with filler volume content $(\varphi)$ follows the below scaling law near the percolation threshold $(\varphi c)$ :

$$
\sigma \propto \sigma_{0}\left(\varphi-\varphi_{c}\right)^{v}
$$

Where oo is the bulk electrical conductivity of the fillers andu is the critical exponent describing the rapid variation of $\sigma$ near $\varphi c$. The percolation threshold is the critical content above which a continuous connected network is formed for the transport of electrons throughout the matrix. The inset in figure. 5 shows the double-logarithmic plot of electrical conductivity of PET/graphene nanocomposites versus $(\varphi-\varphi c)$, and an "anomalous" critical exponent (4.22) was obtained from the slope of linear fit curve, much larger than the theoretically predicted value $(\sim 2)$ for three-dimensional system (Balberg, 1987).

To predict the electrical percolation threshold in theory, filler shape must be first considered in view of its strong influence on the electrical properties of composites (Ma \& Gao, 2008). However, the electrical percolation threshold cannot be directly related to filler morphology, fortunately, geometrical percolation threshold (pc) provides some insight into the former although cannot be simply identified as it. Therefore, several models were developed to explore the percolative properties of oblate objects in respect of their aspect ratio (Ambrosetti et al., 2008; Garboczi et al., 1995; Yi \& Tawerghi, 2009). Recently, Ambrosetti and coworkers (Ambrosetti et al., 2008) proposed a model consisting of isotropically distributed hard oblate spheroids of identical dimensions with soft shell, more fits the system of conducting spheroids dispersed in an insulating continuum host, and the shell thickness can be interpreted as a typical tunneling length between the particles, governing the electrical connectivity of composite. The percolation threshold, pc, for infinite system was obtained by extrapolating that for finite systems through the following scaling relations (Rintoul \& Torquato, 1997):

$$
\begin{gathered}
\Delta(L) \propto L^{-1 / \delta} \\
p_{c}^{e f f}-p_{\mathrm{c}} \propto L^{-1 / \delta}
\end{gathered}
$$

where $\delta$ is the correlation length exponent, $\Delta$ represents the width of the percolation transition, pceff is the effective percolation threshold for a system of linear size L. The value of correlation exponent $\delta$ is calculated as around 0.9 from thousands of simulations for variously sized lattices, and then pc can be consequently estimated from Eq. (4) by plotting pceff against $L-\delta$ for selected values of cell size L. The simulation results, for oblate ellipsoids with various aspect ratio and penetrable shell thickness, indicated that high aspect ratio entails a lower percolation threshold and this effect is also dependant on the shell 
thickness, consistent with the behavior observed in our study and other composites containing oblate objects as conducting fillers. Therefore, the small $\varphi c$ of PET/graphene nanocomposites mainly owes to the high aspect ratios of graphene nanosheets.

Moreover, if neglecting the limiting interaction distances to a shell of constant thickness from the presence of the hard core, the model will evolve to an idealized model consisting of completely permeable ellipsoids randomly dispersed in a matrix, as Garboczi and coworkers (Garboczi et al., 1995) suggested. From this model, pc can be predicted as 1.27 $\alpha / \beta$ where $\beta$ is the radius of revolution and $\alpha$ is the semiaxis length in the extreme oblate limit. Therefore, pc can be easily calculated as $0.87 \mathrm{vol} \%$ for PET/graphene nanocomposites and $3.34 \mathrm{vol} \%$ for PET/graphite composites if graphene and graphite considered as permeable oblate particles. These values are higher than that of $\varphi c$ obtained experimentally from curves of Figure 5. This difference between pc and $\varphi c$ may be, in part, due to the assumption that graphite and graphene were permeable, and in part, to the fact that Eq. (2) did not consider tunneling mechanism. The permeable assumption allows regions of space to be occupied by parts of more than one sheet and thereby the critical volume of fillers is overestimated. In addition, the unfavorable geometries of oblate particles also tend to reduce the interparticle connectivity and increase the percolation point. Finally, it is noticed that physically contacted network is adequate but not absolutely necessary for current flow in insulator-conductor composites, since electrons can transmit between isolated particles by tunneling through thin polymer layer (Quivy et al., 1989; Toker et al., 2003).

From above experimental results, it is can be seen that the efficiency of graphene in improving conductivity of PET is comparable to or even better than CNTs. Hu (Hu et al., 2006) reported a low electrical percolation threshold $(0.9 \mathrm{wt} \%)$ in PET/MWCNT nanocomposites fabricated via coagulation, and the electrical conductivity reached $10-2 \mathrm{~S} / \mathrm{m}$ at higher filler loading $(\sim 5 \mathrm{wt} \%)$. A small percolation threshold was reported by Steinert and coworkers in PET/SWCNT nanocomposite films prepared using a solution mixing and casting method. It was shown that sufficient conductivity for antistatic and electrostatic dissipation purposes was achieved at $0.5 \mathrm{wt} \%$ SWCNT, which was easily understood because solution casting of a film is efficient to form a conductive network due to the 2dimentional distribution of CNT and less serious damage of CNT length. However, melt compounding like twin-screw extrusion would inevitably shorten CNT and thus reduce its aspect ratio, which is not beneficial for achieving small percolation thresholds and high conductivity. For example, CNTs-based composites prepared by melt blending with coupling agent only leads to a moderate conductivity of $0.1 \mathrm{~S} / \mathrm{m}$ with $2.48 \mathrm{vol} \%$ CNTs (Li et al., 2006). Fortunately, the electrical percolation threshold ( $\varphi c=0.47 \mathrm{vol} \%)$ obtained in this study is also one of the lowest values for graphite-based composites (Chen et al., 2003; Zheng \& Wong, 2003) although slightly higher than that observed in polystyrene/graphene composites prepared by solution mixing (Stankovich et al., 2006). This low percolation threshold and the good conductivity are attributed to the high aspect ratio, large specific surface area and the good dispersion of graphene in PET matrix.

In addition, we found the anomalous exponent of 4.22 for the PET/graphene nanocomposites. Indeed, nonuniversal values $(\geq 3)$ of the critical exponent were reported in many systems (Celzard et al., 1996; Carmona et al., 1984; Ezquerra et al., 1990). To explain this phenomenon, the tunneling conduction mechanism is repeatedly proposed. Balberg proposed a model based on the interparticle tunneling in percolation network. The conductance distribution function of the network, $\mathrm{f}(\mathrm{g})$, was related to the tunneling 
conductance, $\mathrm{g}$, between two impermeable spherical particles with a radius of $\mathrm{b}$ and an average interparticle distance a by:

$$
f(g) \propto \ln \left(g_{0} / g\right) g^{\left(d_{0} / a\right)-1}
$$

where $\mathrm{g}_{0}$ is a constant and $\mathrm{d}_{0}$ is the characteristic distance for tunneling (usually several nanometers). Percolation network and tunneling conductance are two competitive mechanisms in conductive composites, and the configuration of conductive fillers in polymer matrix determines the competition of these two mechanisms. Fillers existing as large and complicated aggregates (i.e. high structured carbon blacks) in composites are easy to cause much narrower distribution of interparticle tunneling distances than that of the distances between two adjacent particles, namely, the case of $\mathrm{d} 0>\mathrm{a}$, for small $g$ values, the conductance function can account for the network and a universal behavior will be obtained (Balberg \& Binenbaum, 1985). Whereas, smaller and less agglomerated filler structure (low structure carbon blacks) can provide a wide-enough distribution of the distances between particle surfaces, $\mathrm{d} 0<\mathrm{a}$, it can be seen that the essential part of the conductance distribution is determined and given by Eq. (6), which is well known to yield a anomalous behavior (Kogut \& Straley, 1979).

$$
g^{\left(d_{0} / a\right)-1} \equiv g^{-a}
$$

Thus, an anomalous critical exponent of 4.0 was found in the composites filled with uniformly dispersed carbon blacks. Notice that the relationship given by Eq. (6) can be extended to general case of $a \geq 2 b$. For our PET/graphene nanocomposites, the evenly dispersed monolayer graphene or very small aggregates would also result in wide distribution of the interparticle distances similar to that derived from the above low structure carbon blacks in polyvinylchloride. Therefore, Eq.s (5) and (6) can be applied to interpret the nonuniversal conductivity in PET/graphene nanocomposites. Additionally, the vast nanosheet-polymer interface provides abundant potential sites for tunneling conductance, thereby an anomalous critical exponent of 4.22 was found in PET/graphene nanocomposites.

The nonuniversal behavior can also be associated with the anisotropy (Smith \& Lobb, 1979) of conductive fillers and the neck-like bonds in the percolation backbone. Both experimental and theoretical results indicate that geometrical and electrical anisotropy degree is expected to influence the percolation threshold and critical exponent.

\section{Conclusions}

Graphene sheets were prepared by chemical oxidation of pristine graphite flakes followed by thermal exfoliation and reduction of graphite oxide. PET/graphene nanocomposites prepared by melt compounding exhibit superior electrical conductivity with a small percolation threshold of $0.47 \mathrm{vol} \%$. A high electrical conductivity of $2.11 \mathrm{~S} / \mathrm{m}$ of PET nanocomposite was achieved with only $3.0 \mathrm{vol} \%$ of graphene, which is even adequate for EMI shielding. Additionally, an anomalous critical exponent of 4.22 for PET/graphene nanocomposites can be ascribed to the tunneling conduction and conductivity anisotropy of graphene. Compared to expensive CNTs, graphene is cheaper and it is more effective in making insulating polymers electrically conductive due to its higher specific surface area than CNTs. 


\section{References}

Alig I., Lellinger D., Dudkin S.M. \& Potschke P. (2007). Polymer, 48(4):1020-1029.

Ambrosetti G., Johner N., Grimaldi C., Danani A. and Ryser P. (2008). Phys Rev E, 78(6), 061126.

Balandin A.A., Ghosh S., Bao W.Z., Calizo I., Teweldebrhan D., Miao F. \& Lau C.N. (2008). Nano Letters, 8(3):902-907.

Balberg I. \& Binenbaum N. (1985). Phys Rev A, 31(2):1222-1225.

Balberg I. (1987). Phys Rev Lett, 59(12):1305-1308.

Berger C., Song Z.M., Li T.B., Li X.B., Ogbazghi A.Y., Feng R., Dai Z.T., Marchenkov A.N., Conrad E.H., First P.N. \& de Heer W.A. (2004) JPhys Chem B, 108(52):19912-19916.

Carmona F., Prudhon P. \& Barreau F. (1984). Solid State Commun, 51(4):255-257.

Carlsson J.M. (2007). Nature Materials, 6(11):801-802.

Celzard A., Furdin G., Mareche J.F., McRae E., Dufort M. \& Deleuze C. (1994). Solid State Commun, 92(5):377-383.

Celzard A.M.E., Mareche J.F., Furdin G., Dufort M. \& Deleuze C. (1996). JPhys Chem Solids, 57(6-8):715-718.

Chen G.H., Weng W.G., Wu D.J. \& Wu C.L. (2003). Eur Polym J, 39(12):2329-2335.

Cobbs W.H. \& Burton R.L. (1953). JPolym Sci, 10(3):275-290.

Ezquerra T.A., Kulescza M., Cruz C.S. \& Baltacalleja F.J. (1990). Adv Mater, 2(12): 597-600.

Garboczi E.J., Snyder K.A., Douglas J.F. \& Thorpe M.F. (1995). Phys Rev E, 52(1):819-828.

Grossiord N., Loos J. \& Koning C.E. (2005). JMater Chem, 15(24):2349-2352.

Halperin B.I., Feng S. \& Sen P.N. (1985). Phys Rev Lett, 54(22):2391-2394.

Heersche H.B., Jarillo-Herrero P., Oostinga J.B., Vandersypen L.M.K. \& Morpurgo A.F. (2007). Nature, 446(7131):56-59.

Hu G.J., Zhao C.G., Zhang S.M., Yang M.S. \& Wang Z.G. (2006). Polymer, 47(1):480-488.

Kalaitzidou K., Fukushima H. \& Drzal L.T. (2007). Carbon, 45(7):1446-1452.

Kelly B. (1981). Physics of graphite, London Englewood N.J: Applied science publishers, pp. 267-361.

Kim H. \& Macosko C.W. (2008). Macromolecules, 41(9):3317-3327.

Kogut P.M. \& Straley J.P. (1979). JPhys C- Solid State Phys, 12(11):2151-2159.

Lagreve C. Feller J.F., Linossier I. \& Levesque G. (2001). Polym Eng Sci, 41(7): 1124-1132.

Lee C., Wei X.D., Kysar J.W. \& Hone J. (2008). Science, 321(5887):385-388.

Li Z.F., Luo G.H., Wei F. \& Huang Y. (2006). Compos Sci Technol, 66(7-8):1022-1029.

Liang J.J., Wang Y., Huang Y., Ma Y.F., Liu Z.F., Cai F.M., Zhang C.D., Gao H.J. \& Chen Y.S. (2009). Carbon, 47(3):922-925.

Ma H.M. \& Gao X.L. (2008). Polymer, 49(19):4230-4238.

McAllister M.J., Li J.L., Adamson D.H., Schniepp H.C., Abdala A.A., Liu J., Herrera-Alonso M., Milius D.L., Car R., Prud'homme R.K. \& Aksay I.A. (2007). Chem Mater, 19(18): 4396-4404.

McLachlan D.S., Chiteme C., Park C., Wise K.E., Lowther S.E., Lillehei P.T., Siochi E.J. \& Harrison J.S. (2005). JPolym Sci Pol Phys, 43(22):3273-3287.

Meyer J.C. Geim A.K., Katsnelson M.I., Novoselov K.S., Booth T.J. \& Roth S. (2007). Nature, 446(7131):60-63.

Novoselov K.S., Geim A.K., Morozov S.V., Jiang D., Zhang Y., Dubonos S.V., Grigorieva I.V. \& Firsov A.A. (2004). Science, 306(5696):666-669. 
Novoselov K.S., Geim A.K., Morozov S.V., Jiang D., Katsnelson M.I., Grigorieva I.V., Dubonos S.V. \& Firsov A.A. (2005). Nature, 438(7065):197-200.

Novoselov K.S., Jiang Z., Zhang Y., Morozov S.V., Stormer H.L., Zeitler U., Maan J.C., Boebinger G.S., Kim P. \& Geim A.K. (2007). Science, 315(5817):1379-1379.

Peigney A, Laurent C, Flahaut E, Bacsa RR, \& Rousset A. Carbon, 2001; 39(4): 507-514.

Quivy A., Deltour R., Jansen A.G.M. \& Wyder P. (1989). Phys Rev B, 39(2):1026-1030.

Ramanathan T., Abdala A.A., Stankovich S., Dikin D.A., Herrera-Alonso M., Piner R.D., Adamson D.H., Schniepp H.C., Chen X., Ruoff R.S., Nguyen S.T., Aksay I.A., Prud'homme R.K. \& Brinson L.C. (2008). Nature Nanotechnology, 3(6):327-331.

Rintoul M.D. \& Torquato S. (1997). JPhys A-math Gen, 30(16):L585-L592.

Schniepp H.C., Li J.L., McAllister M.J., Sai H., Herrera-Alonso M., Adamson D.H., Prud'homme R.K., Car R., Saville D.A. \& Aksay I.A. (2006). JPhys Chem B, 110(17): 8535-8539.

Smith L.N. \& Lobb C.J. (1979). Phys Rev B, 20(9):3653-3658.

Stankovich S., Dikin D.A., Dommett G.H.B., Kohlhaas K.M., Zimney E.J., Stach E.A., Piner R.D., Nguyen S.T. \& Ruoff R.S. (2006). Nature, 442(7100):282-286.

Staudenmaier L. (1898). Ber Dtsch Chem Ges, 31:1481-1499.

Stauffer D. (1985). An introduction to percolation theory, Landon and Philadephia: Taylor and Fansis, 325-355.

Steinert B.W. \& Dean D.R. (2009). Polymer, 50(3):898-904.

Toker D., Azulay D., Shimoni N., Balberg I. \& Millo O. (2003). Phys Rev B, 68: 041403.

Viculis L.M., Mack J.J. \& Kaner R.B. (2003). Science, 299(5611):1361-1361.

Wang G.X., Yang J., Park J., Gou X.L., Wang B., Liu H. \& Yao J. (2008). JPhys Chem C, 112(22):8192-8195.

Wang X.B., You H.J., Liu F.M., Li M.J., Wan L., Li S.Q., Li Q., Xu Y., Tian R., Yu Z.Y., Xiang D. \& Cheng J. (2009). Chem Vapor Depos, 15(1-3):53-56.

Weng W.G., Chen G.H. \& Wu D.J. (2005). Polymer, 46(16):6250-6257.

Xie S.H., Liu Y.Y. \& Li J.Y. (2008). Appl Phys Lett, 92(24):243121.

Yao S.H., Dang Z.M., Jiang M.J. \& Xu H.P. (2007). Appl Phys Lett, 91(21):042914.

Yi Y.B. \& Tawerghi E. (2009). Phys Rev E, 79: 041134.

Zhang M., Li D.J., Wu D.F., Yan C.H., Lu P. \& Qiu G.M. (2008). JAppl Polym Sci, 108(3):14821489.

Zhang Y.B., Tan Y.W., Stormer H.L. \& Kim P. (2005). Nature, 438(7065):201-204.

Zhang Y.B., Small J.P., Amori M.E.S. \& Kim P. (2005). Phys Rev Lett, 94(17): 178603.

Zheng W.G., Wong S.C. \& Sue H.J. (2002). Polymer, 43(25):6767-6773.

Zheng W. \& Wong S.C. (2003). Compos Sci Technol, 63(2):225-235. 


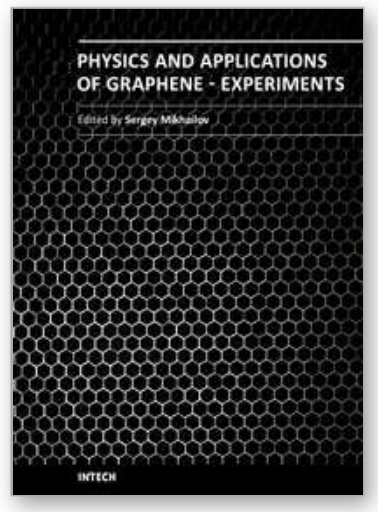

\author{
Physics and Applications of Graphene - Experiments \\ Edited by Dr. Sergey Mikhailov
}

ISBN 978-953-307-217-3

Hard cover, 540 pages

Publisher InTech

Published online 19, April, 2011

Published in print edition April, 2011

The Stone Age, the Bronze Age, the Iron Age... Every global epoch in the history of the mankind is characterized by materials used in it. In 2004 a new era in material science was opened: the era of graphene or, more generally, of two-dimensional materials. Graphene is the strongest and the most stretchable known material, it has the record thermal conductivity and the very high mobility of charge carriers. It demonstrates many interesting fundamental physical effects and promises a lot of applications, among which are conductive ink, terahertz transistors, ultrafast photodetectors and bendable touch screens. In 2010 Andre Geim and Konstantin Novoselov were awarded the Nobel Prize in Physics "for groundbreaking experiments regarding the two-dimensional material graphene". The two volumes Physics and Applications of Graphene - Experiments and Physics and Applications of Graphene - Theory contain a collection of research articles reporting on different aspects of experimental and theoretical studies of this new material.

\title{
How to reference
}

In order to correctly reference this scholarly work, feel free to copy and paste the following:

Haobin Zhang, Shunlun He, Cao Chen, Wenge Zheng and Qing Yan (2011). Electrical Conductivity of Melt Compounded Functionalized Graphene Sheets Filled Polyethyleneterephthalate Composites, Physics and Applications of Graphene - Experiments, Dr. Sergey Mikhailov (Ed.), ISBN: 978-953-307-217-3, InTech, Available from: http://www.intechopen.com/books/physics-and-applications-of-grapheneexperiments/electrical-conductivity-of-melt-compounded-functionalized-graphene-sheets-filledpolyethyleneterepht

\section{INTECH}

open science | open minds

\section{InTech Europe}

University Campus STeP Ri

Slavka Krautzeka 83/A

51000 Rijeka, Croatia

Phone: +385 (51) 770447

Fax: +385 (51) 686166

www.intechopen.com

\section{InTech China}

Unit 405, Office Block, Hotel Equatorial Shanghai

No.65, Yan An Road (West), Shanghai, 200040, China 中国上海市延安西路65号上海国际贵都大饭店办公楼405单元

Phone: +86-21-62489820

Fax: +86-21-62489821 
(C) 2011 The Author(s). Licensee IntechOpen. This chapter is distributed under the terms of the Creative Commons Attribution-NonCommercialShareAlike-3.0 License, which permits use, distribution and reproduction for non-commercial purposes, provided the original is properly cited and derivative works building on this content are distributed under the same license. 